\title{
Rolling induced size effects in elastic-viscoplastic sheet metals
}

\author{
Nielsen, Kim Lau
}

Published in:

European Journal of Mechanics A - Solids

Link to article, DOI:

10.1016/j.euromechsol.2015.05.006

Publication date:

2015

Document Version

Peer reviewed version

Link back to DTU Orbit

Citation (APA):

Nielsen, K. L. (2015). Rolling induced size effects in elastic-viscoplastic sheet metals. European Journal of Mechanics A - Solids, 53, 259-267. https://doi.org/10.1016/j.euromechsol.2015.05.006

\section{General rights}

Copyright and moral rights for the publications made accessible in the public portal are retained by the authors and/or other copyright owners and it is a condition of accessing publications that users recognise and abide by the legal requirements associated with these rights.

- Users may download and print one copy of any publication from the public portal for the purpose of private study or research.

- You may not further distribute the material or use it for any profit-making activity or commercial gain

- You may freely distribute the URL identifying the publication in the public portal

If you believe that this document breaches copyright please contact us providing details, and we will remove access to the work immediately and investigate your claim 


\title{
Rolling induced Size effects in Elastic-viscoplastic Sheet Metals
}

\author{
K.L. Nielsen* \\ Department of Mechanical Engineering, Solid Mechanics, Technical University of Denmark, \\ DK-2800 Kgs. Lyngby, Denmark
}

\begin{abstract}
Rolling processes for which the characteristic length scale reaches into the range where size effects become important are receiving increased interest. In particularly, this is owed to the roll-molding process under development for high-throughput of micronscale surface features. The study presented revolves around the rolling induced effect of visco-plasticity (ranging hot and cold rolling) in combination with strain gradient hardening - including both dissipative and energetic contributions. To bring out first order effects on rolling at small scale, the modeling efforts are limited to flat sheet rolling, where a non-homogeneous material deformation takes place between the rollers. Large strain gradients develop where the rollers first come in contact with the sheet, and a higher order plasticity model is employed to illustrate their influence at small scales. The study reveals that the energetic length parameter has negligible effect on the rolling quantities of interest, while the contribution coming from the dissipative length parameter can be dominant. Considering a slow and a fast moving sheet, respectively, convergence towards the rate independent limit is demonstrated, and a characteristic velocity is identified, for which the torque and punch force applied to the roller becomes independent of the material rate-sensitivity.
\end{abstract}

\footnotetext{
${ }^{*}$ Tel: +45 4525-4258, Fax: +45 4593-1475

URL: kin@mek.dtu.dk (K.L. Nielsen)
} 
Keywords: Higher order theory, Metal forming, Rolling, Foils

\section{Introduction}

Rolling at small scale has recently received attention due to an apparent size effects observed when down-scaling experiments. One suspect to this is strain gradient hardening. It is well-established that with large plastic strain gradients come an increased hardening at micron scale - and rolling is no exception. As the sheet is forced between the rollers, a fairly heterogeneous evolution of plastic straining takes place and severe gradients develop (Richelsen, 1993, 1996). To accommodate the large plastic gradients, Geometrically Necessary Dislocations (GNDs) are forced to develop, and with the movement and storage of these additional dislocations come added free energy and dissipation (Ashby, 1970; Gurtin, 2002; Ohno and Okumara, 2007). At micron scale, GNDs can become a substantial portion of the total dislocation density, and thus dominate the amount of energy required to deform the material.

Nielsen et al. (2015) recently demonstrated rolling related size effects in the rateindependent limit of an elastic-viscoplastic solid (cold rolling), employing a steady-state numerical framework. By accounting for a dissipative length scale, it has been shown that the forces (punch force, roll torque, power input etc.), and hence the contact interface conditions, between the rollers and the sheet, generally displays increased levels. As discussed by Richelsen (1991), modeling the rolling process in a traditional Lagrangian finite element framework is by no means trivial. E.g. numerical issues arise when taking into account the continuously changing contact interface as the region moves relative to the discretized domain when the material passes between the rollers. In addition, complexity is added by the frictional stresses changing direction within a 
narrow sticking region for which the size and position is unknown in advance. All of these numerical issues are avoided in a steady-state framework by letting the discretized domain remain stationary relative to the rollers, while the sheet material passes through the domain. Thus, the contact interface, sticking region, and stress/strain fields become stationary to an observer at the rollers. This is all about relative motion, but the stationarity makes the numerical task easier to tackle.

Numerous numerical investigations of the rolling process have been undertaken and count both 1D, 2D and 3D studies (Montmitonnet, 2006). A large portion of these accepts rigid-plasticity or visco-plasticity as an approximation (Zienkiewicz et al., 1978; Mori et al., 1982; Cavaliere et al., 2001), and residual stresses and the associate material behaviour are typically neglected. Sheet rolling often takes place at elevated temperatures (hot rolling), ranking material sensitivity essential, but the importance of elastic unloading is recognized for rolling at room temperature (cold rolling). The steady-state formulation put forward by Dean and Hutchinson (1980) is well suitable for history dependent material deformation processes and it readily accounts for elastic unloading. Their method has been adapted to rolling in the study by Nielsen et al. (2015), and it will be further exploited in the present investigation. The objective of the present study is to gain insight into, and quantify, the combined effect of strain ratesensitivity and strain gradient hardening during flat sheet rolling; essentially studying rate effects as the characteristic length scale reaches into the range where size effects become important. Both dissipative and energetic contributions are included.

The paper is structured as follows. The material model and steady-state formulation are presented in Section 2, while the considered boundary value problem is outlined in 
Section 3. Results are laid out in Section 4 and discussed with focus on the combined effect of strain rate-sensitivity and strain gradient hardening. Some concluding remarks are given in Section 5 .

\section{Model: constitutive relations and steady-state formulation}

\subsection{Rate-sensitive constitutive material model}

The flat rolling problem is analyzed using the gradient enhanced elastic-viscoplastic material model proposed in Gudmundson (2004); Gurtin and Anand (2005); Fleck and Willis (2009). Here, a small strain formulation is employed. This is a reasonable approximation to the rolling process as the overall straining is proportional to the sheet reduction when limiting this to $\sim 15 \%$. For small sheet reductions, the strains and the rotations remain small - yet large plastic strain gradients can evolve (see e.g. Fig. 6). An additive decomposition of the total strain is applied, so that $\varepsilon_{i j}=\varepsilon_{i j}^{e}+\varepsilon_{i j}^{p}$, where $\varepsilon_{i j}^{e}$ is the elastic part and $\varepsilon_{i j}^{p}$ is the plastic part. The total strain field is determined from the displacements, which together with the plastic strain components are determined based on the principle of virtual work for the current higher order material. In Cartesian components, this reads

$$
\int_{V}\left(\sigma_{i j} \delta \varepsilon_{i j}+\left(q_{i j}-s_{i j}\right) \delta \varepsilon_{i j}^{p}+\tau_{i j k} \delta \varepsilon_{i j, k}^{p}\right) \mathrm{d} V=\int_{S}\left(T_{i} \delta u_{i}+M_{i j} \delta \varepsilon_{i j}^{p}\right) \mathrm{d} S .
$$

where $q_{i j}$ is the micro-stress tensor, $\sigma_{i j}$ is the Cauchy stress tensor, $s_{i j}=\sigma_{i j}-\delta_{i j} \sigma_{k k} / 3$ is the stress deviator and $\tau_{i j k}$ is the higher order stresses, work conjugate to the plastic strain gradients, $\varepsilon_{i j, k}^{p}$. Here, ()$_{, k}$ denotes the partial derivative with respect to the coordinate $x_{k}$. The right-hand side of Eq. (1) includes both conventional tractions, 
$T_{i}=\sigma_{i j} n_{j}$, and higher order tractions, $M_{i j}=\tau_{i j k} n_{k}$, with $n_{k}$ denoting the outward normal to the surface $S$, which bounds the volume $V$.

Following Fleck and Willis (2009), the higher order stresses decompose into a dissipative part, $\tau_{i j k}^{D}$, and a energetic part, $\tau_{i j k}^{E}$, so that; $\tau_{i j k}=\tau_{i j k}^{D}+\tau_{i j k}^{E}$, whereas the micro-stress is assumed to have a dissipative part; $q_{i j}=q_{i j}^{D}$, only. The dissipative stress quantities read (Gudmundson, 2004; Fleck and Willis, 2009)

$$
q_{i j}^{D}=\frac{2}{3} \frac{\sigma_{C}\left[\dot{E}^{p}, E^{p}\right]}{\dot{E}^{p}} \dot{\varepsilon}_{i j}^{p}, \quad \text { and } \quad \tau_{i j k}^{D}=\frac{\sigma_{C}\left[\dot{E}^{p}, E^{p}\right]}{\dot{E}^{p}}\left(L_{D}\right)^{2} \dot{\varepsilon}_{i j, k}^{p}
$$

with the gradient enhanced effective stress identified as; $\sigma_{C}=\sqrt{\frac{3}{2} q_{i j}^{D} q_{i j}^{D}+\left(L_{D}\right)^{-2} \tau_{i j k}^{D} \tau_{i j k}^{D}}$. The corresponding gradient enhanced effective plastic strain rate takes a quadratic form, so that

$$
\dot{E}^{p}=\sqrt{\frac{2}{3} \dot{\varepsilon}_{i j}^{p} \dot{\varepsilon}_{i j}^{p}+\left(L_{D}\right)^{2} \dot{\varepsilon}_{i j, k}^{p} \dot{\varepsilon}_{i j, k}^{p}}
$$

where, $L_{D}$ is the dissipative length parameter introduced for dimensional consistency.

Plastic deformations are typically considered to be dissipative, covering irrecoverable heat energy and cold work, while no free energy is associated with the plastic strains. However, when large plastic strain gradients appear (Ashby, 1970), Geometrically Necessary Dislocations (GNDs) are develop, and this gives rise to additional free energy associated with the local stress field of the GNDs, as-well as increased dissipation when the GNDs move in the lattice (Gurtin, 2002; Ohno and Okumara, 2007). Thus, the total free energy takes the form

$$
\Psi=\frac{1}{2}\left(\varepsilon_{i j}-\varepsilon_{i j}^{p}\right) \mathcal{L}_{i j k l}\left(\varepsilon_{k l}-\varepsilon_{k l}^{p}\right)+\Psi_{G}
$$

where $\Psi_{G}$ accounts for the free energy associated with GNDs. The conventional stresses is, thereby, given through the elastic relation; $\sigma_{i j}=\partial \Psi / \partial \varepsilon_{i j}^{e}=\mathcal{L}_{i j k l}\left(\varepsilon_{k l}-\varepsilon_{k l}^{p}\right)$, with $\mathcal{L}_{i j k l}$ 
being the isotropic elastic stiffness tensor, while the energetic higher order stresses are defined as; $\tau_{i j k}^{E}=\partial \Psi / \partial \varepsilon_{i j, k}^{p}$. The free energy related to GNDs are often assumed to be quadratic, with respect to the plastic strain gradients, so that; $\Psi_{G}=\frac{1}{2} G\left(L_{E}\right)^{2} \varepsilon_{i j, k}^{p} \varepsilon_{i j, k}^{p}$. Thus the energetic higher order stresses read; $\tau_{i j k}^{E}=G\left(L_{E}\right)^{2} \varepsilon_{i j, k}^{p}$, where $G$ is the elastic shear modulus and $L_{E}$ is the energetic length parameter. This setup is employed throughout the present study, but it is recognized that an on-going discussion of the form of $\Psi_{G}$ takes place in the literature (Fleck et al., 2015). In any case, it will become evident from the results that the energetic contribution has a negligible effect on sheet rolling.

The developed model rely on a power-law relation for the visco-plastic behaviour, so that

$$
\dot{E}^{p}=\dot{\varepsilon}_{0}\left(\frac{\sigma_{C}}{g\left(E^{p}\right)}\right)^{1 / m}, \quad \text { with } \quad g\left(E^{p}\right)=\sigma_{y}\left(1+\frac{E E^{p}}{\sigma_{y}}\right)^{N}
$$

where $N$ is the power hardening exponent, $m$ is the strain rate hardening exponent and $\dot{\varepsilon}_{0}$ is the reference strain rate. Thus, $\sigma_{C}\left[E^{p}, \dot{E}^{p}\right]=g\left(E^{p}\right)\left(\dot{E}^{p} / \dot{\varepsilon}_{0}\right)^{m}$. Thus, the developed model display significant visco-plastic behaviour for large strain rate hardening exponents, but approaches the response of a gradient enhanced J2-flow type material in the rate-independent limit $(m \rightarrow 0$, see e.g. Fleck and Willis, 2009; Nielsen and Niordson, 2013, 2014). Moreover, the response of the gradient enhanced model reduces to the prediction of its corresponding conventional version for zero length scales $\left(L_{D}=L_{E}=0\right)$. The numerical framework is, however, unstable in the limit when $L_{D} \rightarrow 0$, and thus a conventional material model has been independently developed and coded for comparison in Nielsen et al. (2015). The material properties considered in the present study are summarized in Tab. 1. 


\subsection{Steady-State Formulation}

The steady-state finite element (FE) formulation proposed by Dean and Hutchinson (1980) is chosen over a classical transient Lagrangian modeling approach since it directly brings out the steady-state field that appears stationary relative to the rollers. Thus, convergence issues of any transient behaviour are avoided, making the steadystate formulation more precise and less demanding in terms of calculation time (see also Hacquin et al. (1996) for a similar approach). Moreover, the modeling approach directly accommodates elastic-plastic unloading, and can be adapted to a wide range of constitutive models.

Dean and Hutchinson (1980) originally define steady-state in the context of crack propagation as the condition for which the stress/strain field surrounding an advancing crack tip remains unchanged to an observer moving with the tip. A similar approach can be adopted for the rolling process, where stationarity of the stress/strain field must exist for a continuous feed of a homogeneous sheet. Thus, the stress/strain field remains unchanged to an observer at the rollers seeing the material pass by. Any time derived quantity, $\dot{f}$, in the constitutive model can thereby be related to the spatial derivative through the sheet velocity, $\dot{a}$, along the $x_{1}$-direction, according to $\dot{f}=-\dot{a} \frac{\partial f}{\partial x_{1}}$. An incremental quantity, at a given material point $\left(x_{1}^{*}, x_{2}^{*}\right)$, can thereby be evaluated by a streamline integration along the negative $x_{1}$-direction (see Fig. 1), which starts well in front of the active plastic zone (upstream, $x_{1}=x_{1}^{0}>>0, x_{2}=x_{2}^{*}$ ) and ends at the point of interest $\left(x_{1}=x_{1}^{*}, x_{2}=x_{2}^{*}\right)$. E.g. the plastic strains are determined as;

$$
\varepsilon_{i j}^{p}\left(x_{1}^{*}, x_{2}^{*}\right)=\int_{x_{1}^{0}}^{x_{1}^{*}} \frac{\partial \varepsilon_{i j}^{p}}{\partial x_{1}} \mathrm{~d} x_{1}, \quad \text { with } \quad \frac{\partial \varepsilon_{i j}^{p}}{\partial x_{1}}=-\frac{1}{\dot{a}} \dot{\varepsilon}_{i j}^{p}
$$


and $\dot{\varepsilon}_{i j}^{p}$ being the plastic strain rates. The spatial streamline integration is carried out using a standard forward Euler time integration, with the point of interest holding the history of all upstream material points.

In the chosen model formulation, the conventional principle of virtual work for quasi-static problems can be use to determine the displacement field, $u_{i}$ (which corresponds to Minimum Principle II in Fleck and Willis, 2009),

$$
\int_{V} \mathcal{L}_{i j k l} \varepsilon_{k l} \delta \varepsilon_{i j} \mathrm{~d} V=\int_{S} T_{i} \delta u_{i} \mathrm{~d} S+\int_{V} \mathcal{L}_{i j k l} \varepsilon_{k l}^{p} \delta \varepsilon_{i j} \mathrm{~d} V
$$

whereas a corresponding minimum principle can be formulated for the plastic strain rate field (which corresponds to Minimum Principle I in Fleck and Willis, 2009),

$$
\int_{V}\left(q_{i j}^{D} \delta \dot{\varepsilon}_{i j}^{p}+\tau_{i j k}^{D} \delta \dot{\varepsilon}_{i j, k}^{p}\right) \mathrm{d} V=\int_{V}\left(s_{i j} \delta \dot{\varepsilon}_{i j}^{p}-\tau_{i j k}^{E} \delta \dot{\varepsilon}_{i j, k}^{p}\right) \mathrm{d} V+\int_{S} M_{i j} \delta \dot{\varepsilon}_{i j}^{p} \mathrm{~d} S .
$$

The displacement field, and the related plastic strain rate field can thereby be iterated upon in a "staggered" approach.

The numerical implementation follows that of Niordson and Hutchinson (2011). Thus, based on the Minimum Principles in Eqs. (7)-(8), a standard finite element interpolation of the form

$$
\dot{u}_{i}=\sum_{n=1}^{8} N^{(n)} \dot{u}_{i}^{(n)} \quad \text { and } \quad \dot{\varepsilon}_{i j}^{p}=\sum_{n=1}^{4} N^{(n)} \dot{\varepsilon}_{i j}^{p(n)}
$$

can be introduced for the displacement increments and the plastic strain rate field, respectively. Here, 8-node isoparametric plane strain elements are used for the discretization of the displacement field, and corresponding 4-node elements are used for the plastic strain rate field. Both element types are integrated using Gauss quadrature, with $2 \times 2$ Gauss points. The nodal solution is iterated upon following a steady-state integration procedure similar to that in Dean and Hutchinson (1980); Niordson (2001); 
Wei and Hutchinson (1997); Nielsen and Niordson (2012). A detailed overview of the algorithm can be found in Nielsen et al. (2012).

\section{Problem formulation and contact procedure}

In steady-state plane strain rolling the translational displacement of the sheet is large, but the strains remain small for moderate thickness reductions. E.g. let the position of a material point at time $t=0$ be $\left(x_{1}, x_{2}\right)$ in the coordinate system defined in Fig. 1 , and denote the position of this same material point at time $t$ by $\left(X_{1}, X_{2}\right)$. The displacements, $u_{i}\left(x_{1}, x_{2}\right)$, used in the formulation are defined by the relation of these two positions of the same material point:

$$
X_{1}=x_{1}-\dot{a} t+u_{1}, \quad X_{2}=x_{2}+u_{2}
$$

where $\dot{a}$ is the constant velocity in the negative $x_{1}$-direction of the undeformed sheet feeding into the rollers. The displacements, $u_{i}\left(x_{1}, x_{2}\right)$, are relative to a frame translating with the feed velocity of the sheet, so that the strains are: $\varepsilon_{i j}=\left(u_{i, j}+u_{j, i}\right) / 2$. Each iteration in the process to solve for the displacements, strains and plastic strains in the current model set-up involves two sequential steps: i) solving for the distribution of plastic strain rates, $\dot{\varepsilon}_{i j}^{p}$, using Minimum Principle I in Eq. (8) and obtaining the plastic strains by integrating along the streamlines as in Eq. (6); and ii) using Minimum Principle II in Eq. (7) to obtain the displacements and total strains.

The thickness of the undeformed sheet feeding into the rollers is $2 H$. The two circular cylindrical rollers of radius, $R$, are considered to be rigid and the downward displacement, $\Delta$, of the upper roller is the depth of the minimum point on the roller surface below $x_{2}=H$ (see Fig. 1). The lower roller is assumed to be displaced upward by the 
same amount, thus symmetry conditions are enforced along $x_{2}=0$ with $u_{2}\left(x_{1}, 0\right)=0$ and $\dot{\varepsilon}_{12}^{p}\left(x_{1}, 0\right)=0$, such that only the upper half of the sheet is meshed. The prescribed punch displacement is $\Delta / H$, which is the thickness reduction, $(H-h) / H$, plus a small elastic spring-back, c.f., Fig. 1. In addition to the material properties and the parameters controlling the rolling configuration $(R / H, \Delta / H)$, a dimensionless feed velocity of $\dot{a} /\left(\dot{\varepsilon}_{0} H\right)$ is also prescribed. Depending on the value of strain rate hardening exponent, a significant influence of the sheet velocity can occur.

To initiate the iterative steady-state procedure, the roller is first punched into the plate while restricting the movement of all nodes that interact with its surface, so that these can only slide along the circular path outlined by the rigid roller. For this, a linear constraint is readily derived, and enforced by a penalty approach. In this way a stress/strain field is created, where after the streamline integration of the constitutive equations can be carried out. In the subsequent iterations, the reaction forces on the roller are continuously checked, and nodes are left free to move in the case a negative pressure on the roller develops.

A "Stick-Slip" condition is employed at the contact interface so that the friction forces obey Coulomb friction whenever slipping occurs, while the material is allowed to stick to the roller whereby the friction forces are set by static equilibrium. Thus, the maximum friction forces that can be transferred between the surfaces are; $F_{f, \max }=$ $\mu F_{n}$, with $\mu$ being the friction coefficient. Sticking is accounted for by imposing the constraint that the straining in the rolling direction (along the $x_{1}$-axis) has to increase monotonically during contact. In case a decrease in straining occurs, the applied nodal friction force will be down-scaled to mimic static friction conditions (with $F_{f, \max }=$ 
$\left.\mu F_{n}\right)$. The down-scaling and adjustment of the friction forces is made continuously during the iterative solution procedure, and allows for a "Sticking" region to evolve (see e.g. Figs. 9-10). Moreover, the blocking of dislocations at the interface between the rollers and the sheet is neglected. Thus, the boundary is free in terms of higher order traction (no constraint on the plastic strains, $\varepsilon_{i j}^{p} \neq 0$ ).

Throughout this study care is taken so that sufficient traction is generated at the interface between the rollers and the sheet top surface for the prescribed constant sheet velocity to be maintained. Thus, no additional pull force will be added to the sheet (see e.g. Nielsen et al., 2015, for details on the procedure). Moreover, no force acts on the sheet in front of the roller. To enforce this condition, a displacement at the right end of the sheet, $u_{1}(L, 0)=\Delta_{A}$, is prescribed and adjusted each iteration such that the reaction force at this point is nearly zero (on the order of $10^{-6}$ of the pull force) in the converged solution. This constraint is enforced solely for numerical reasons and prevents free body motions.

\section{Results}

It has been shown by Nielsen et al. (2015) that the roller radius plays an important role in relation to size effects. Thus, rate-sensitive results will be presented for various roller sizes in the following, while keeping the punch displacement, $\Delta / H$, fixed. Moreover, a friction coefficient of $\mu=0.1$ is employed throughout the study to ensure a constant sheet velocity solely by the action of the rollers (see e.g. Nielsen et al., 2015, for low friction rolling).

As depicted in Figs. 2-3, the evolution of the plastic straining that takes place during flat sheet rolling is largely non-homogeneous. Once the material establish contact with 
the rollers, the plastic straining rapidly increase from the un-deformed state. This increase occurs over a short distance, where after the subsequent increase in straining is moderate, and the sheet eventually leaves the rollers with a fairly homogeneous distribution of plastic strains (see e.g. Fig. 2, or Richelsen, 1993). Comparing Figs. 2 and 3 , it is clear that size effects significantly influence the evolution in the plastic straining. Where large scale rolling displays close lying contours near the point of first contact (hence large gradients, see Fig. 2), much more uniformly spaced strain contours are captured for rolling at small scale (see Fig. 3). This has to do with the penalty that comes with large strain gradients at small scale. As the length parameter increases (corresponding to rolling thinner sheets), a larger amount of energy is needed to deform the material when plastic strain gradients exists. The size effect observed are quantified in Figs. 4-5 for various roller radii, where the influence of the two length parameters $\left(L_{D}\right.$ and $\left.L_{E}\right)$, in combination with visco-plastic effects, are brought out. Focus is on; a) the applied torque, $T$, that the roller transfers to the sheet, and $b$ ) the punch force, $F_{\text {punch }}$, being the required loading on the roller perpendicular to the sheet surface. It is obvious from Fig. 4a that the dissipative length parameter can have a large influence on the material hardening during rolling as the torque applied to the roller increases dramatically - particularly for small roller size. Thus, rolling at small scale (corresponding to large length parameters) requires a relative larger power input ${ }^{1}$ since the storage and movement of the GNDs, needed to accommodate large plastic strain gradients, lead to additional unrecoverable energy tied up in the deformation process.

\footnotetext{
${ }^{1}$ In a small strain setting, the power input is approximated by; $P=|T| \dot{a} / R$, with $T$ being the roll torque, $\dot{a}$ the sheet velocity, and $R$ the roller radius.
} 
On the contrary, the energetic length parameter has a very limited influence on the both the torque and punch force acting on the roller. This has to do with the energetic length parameter being linked to the free energy associated with the current plastic strain gradients (or GND structure). Thus, at steady-state, a change in free energy associated with the rolling process is given by the difference in stored energy between a slice of material sufficiently up-stream and down-stream from the rollers, respectively. By considering rolling of virgin material, zero gradients are found sufficiently far upstream from the rollers, and the residual plastic strain gradients existing down-stream from the rollers are few (see Fig. 6). Hence, the total power input going into the deformation process has to be little affected by the energetic length parameter, which is also indicated by the negligible effect on the roll torque. It is notice from Figs. 4-5 that these observations apply to both high and low sheet velocities independent of the material rate-sensitivity.

Both the dissipative and energetic length parameters only give rise to a small increase in the absolute value of the punch force, while the effect of the material ratesensitivity is significant (see Figs. 4-5). By introducing rate-sensitivity through the strain rate hardening exponent, $m$, the model reveals that both the torque and punch force acting on the roller increase monotonically with increasing rate-sensitivity at high sheet velocity, while a monotonic decrease is found for increasing rate-sensitivity at low sheet velocity. This has to do with the time aspect of the stress build-up/relaxation in the material deformation when $m>0$. At low sheet velocity, the material has time to relax the stress field through plastic straining, whereby the forces (punch and friction force) involved in the rolling process is lowered. Moreover, this visco-plastic 
effect amplifies with increasing $m$-value (see also Eq. (5)). Vice versa, the material has limited time to relax the stress field at high sheet velocity, whereby the roll quantities of interest increase. It is noticed that this response to the rate-sensitivity is similar at all scales (see Figs. 4-5).

Figures 7-8 give away the torque and punch force acting on the roller as function of the normalized sheet velocity, $\dot{a} /\left(\dot{\varepsilon}_{0} H\right)$. What is intriguing here is the fact that all the curves for a given set of length parameters, but with different $m$-values, intersect in a single point that consolidates a characteristic velocity. Thus, the punch force, roll torque, and power input become independent of the material rate-sensitivity at this specific velocity even though the evolution of the plastic deformation can be very different. The existence of the characteristic velocity can be argued according to the visco-plastic stress-relaxation mechanism. Bearing in mind the monotonic behaviour of the roller quantities, and notice their increase with the rate-sensitivity, $m$, for velocities above that intersection point, and vice versa below the intersection point (see Figs. 45). Thus, for two different $m$-values (e.g. $m \rightarrow 0$ and $m=0.2$ ), the curves must necessarily intersect, and as a consequence any curve of intermediate $m$-value intersect at the exact same characteristic velocity.

From Figs. 7-8, it is furthermore seen that an increase in the length parameters (corresponding to rolling at small scale) essentially yields a shift in the curves, while the trends remain the same. The dissipative length parameter is seen to display a significant effect over the entire interval of sheet velocities considered. On the contrary, the influence of the energetic length parameter remains negligible, which is related to the free energy storage/release mechanism discussed above (see e.g. Fig. 6c). 
The contact conditions, at the interface between the roller and the sheet, are illustrated in Figs. 9-10. Here, showing the normalized contact forces (the traction) as function of position, with; $F_{f}^{(e)}$ being the friction force, $F_{n}^{(e)}$ being the normal force, $A^{(e)}$ the element area, and $\sigma_{y}$ the yield stress ${ }^{2}$. Figure 9 displays results for rolling at large scale $\left(L_{D} / H=0.05\right.$ and $L_{E}=0$, resembling the conventional limit), and at two distinct velocities. In the rate-independent limit, the model predictions compare well to previously published results by Richelsen (1996) - both with respect to the level and distribution of the surface traction (see also Nielsen et al., 2015). However, by introducing rate-sensitivity it is easily seen that the stress-relaxation mechanism gives rise to an increase in the general stress level for a fast moving sheet (no time to relax stresses), whereas a somewhat lower level is obtained for a slow moving sheet. This is essentially what is also reflected in the roll torque and punch force discussed previously. Moreover, it is noticed that both the peak traction and the sticking zone slightly shifts with the rate-sensitivity.

The traction distributions found at small scale rolling display close similarities to those at larger scale (compare Figs. 9-10, and see e.g. Nielsen et al., 2015). It is, however, noticed that the contact traction that evolved at small scale is relative higher, and that a somewhat smoother peak exists where the roller first comes in contact with the sheet. This is, in particularly, obvious for large values of the dissipative length parameter (see Fig. 10a). Similarly, the energetic length parameter yields identical trends, but much less pronounced (see Fig. 10b).

\footnotetext{
${ }^{2}$ The chosen configuration of the figures yield the cleanest representation of results, and a comparison with the stress level close to the top boundary of the sheet shows good agreement.
} 


\section{Concluding remarks}

Rolling at small scale is subject to the well-known size effects governed by the evolution of plastic strain gradients. As the sheet is forced between the rollers, the nonhomogeneous deformation gives rise to severe gradients just as first contact between the roller and sheet takes place, whereas a fairly uniform distribution of plastic straining exists through the thickness of the sheet after rolling. Finite element modeling of the rolling process has been undertaken in a steady-state framework, employing the higher order elastic-viscoplastic theory by Fleck and Willis (2009), and the key findings of the study are;

- Rolling at small scale (corresponding to large length parameters) requires a relative larger power input to accommodate the additional unrecoverable energy tied up in the evolution of plastic strain gradients. Throughout, length scale effects have been demonstrated for all roller quantities considered. The dissipative length parameter plays a key role to the forces involved in the process, whereas the energetic length parameter displays a limited effect.

- Depending on the sheet velocity, the material rate-sensitivity yields severe stress build-up/relaxation during rolling at all scales. It is found that the roller quantities of interest (torque, punch forces, power input etc.) increase monotonically for increasing rate-sensitivity at high sheet velocity, while a decrease is found for increasing rate-sensitivity at low sheet velocity (see Figs. 4-5). These findings are directly tied to the predicted changes in the contact conditions, for which similar overall trends are observed (see Figs. 9-10). 
- Based on the monotonic behaviour related to the material rate-sensitivity, a characteristic velocity has been identified for which the predicted roller quantities (torque, punch force, power input etc) becomes independent of the strain rate hardening exponent, $m$, and equals the rate-independent results (see Figs. 7-8). A similar characteristic velocity has been found for steady-state crack propagation in Nielsen and Niordson (2012), and employed in Nielsen et al. (2012) as an approximate way of extracting rate-independent results.

For clarity of results, and to keep focus on the combined effect of material ratesensitivity and strain gradient hardening, the present work takes as off-set the Coulomb friction model. This is an approximation and more complex friction models have been developed for metal forming processes. Adding such complexity is left for future work and the framework developed through the present work lends itself nicely to these studies.

\section{Acknowledgements}

The work is financially supported by The Danish Council for Independent Research under the research career programme Sapere Aude in the project "Higher Order Theories in Solid Mechanics", grant 11-105098/FTP.

\section{References}

Ashby, M., 1970. The deformation of plastically non-homogeneous alloys. Philos. Mag. $21,399-424$.

Cavaliere, M., Goldschmit, M., Dvorkin, E., 2001. Finite element simulation of the steel plates hot rolling process. Int. J. Numer. Methods Engrg. 52, 1141-1430. 
Dean, R., Hutchinson, J., 1980. Quasi-Static Steady Crack Growth in Small-Scale Yielding. Fracture Mechanics: Twelfth Conference, ASTM STP700, American Society for Testing and Materials, 383-405.

Fleck, N., Hutchinson, J., Willis, J., 2015. Guidelines for Constructing Strain Gradient Plasticity Theories. To be published.

Fleck, N., Willis, J., 2009. A mathematical basis for strain-gradient plasticity theory. Part II: Tensorial plastic multiplier. J. Mech. Phys. Solids 57, 1045-1057.

Gudmundson, P., 2004. A unified treatment of strain gradient plasticity. J. Mech. Phys. Solids 52, 1379-1506.

Gurtin, M., 2002. A gradient theory of single-crystal viscoplasticity that accounts for geometrically necessary dislocations. J. Mech. Phys. Solids 50, 5-32.

Gurtin, M., Anand, L., 2005. A theory of strain-gradient plasticity for isotropic, plastically irrotational materials. Part I: Small deformations. J. Mech. Phys. Solids 53, 1624-1649.

Hacquin, A., Montmitonnet, P., Guillerault, J.-P., 1996. A steady state thermoelastoviscoplastic finite element model of rolling with coupled thermo-elastic roll deformation. J. Mater. Process. Techn. 60, 109-116.

Montmitonnet, P., 2006. Hot and cold strip rolling processes. Comp. Meth. in App. Mech. Eng. 193, 16604-6625.

Mori, K., Osakada, K., Oda, T., 1982. Simulation of plane-strain rolling by the rigidplastic finite element method. Int. J. Mech. Sci. 24, 519-527. 
Nielsen, K., Niordson, C., 2012. Rate sensitivity of mixed mode interface toughness of dissimilar metallic materials. Int. J. Solids. Struct. 49, 576-583.

Nielsen, K., Niordson, C., 2013. A 2D finite element implementation of the Fleck-Willis strain-gradient flow theory. Europ. J. Mech. A/Solids 41, 134-142.

Nielsen, K., Niordson, C., 2014. A numerical basis for strain-gradient plasticity theory: rate-independent and rate-dependent formulations. J. Mech. Phys. Solids 63, 113127.

Nielsen, K., Niordson, C., Hutchinson, J., 2012. Strain gradient effects on steady state crack growth in rate-sensitive materials. Eng. Frac. Mech. 96, 61-71.

Nielsen, K., Niordson, C., Hutchinson, J., 2015. Rolling at small scale. (submitted for publication).

Niordson, C., 2001. Analysis of steady-state ductile crack growth along a laser weld. Int. J. Frac. 111, 53-69.

Niordson, C., Hutchinson, J., 2011. Basic strain gradient plasticity theories with application to constrained film deformation. J. Mech. Mater. Struct. 6, 395-416.

Ohno, N., Okumara, D., 2007. Higher-order stress and grain size effects due to selfenergy of geometrically necessary dislocations. J. Mech. Phys. Solids 55, 1879-1898.

Richelsen, A.-B., 1991. Viscoplastic analysis of plane-strain rolling using different friction models. Int. J. Mech. Sci. 33, 761-774.

Richelsen, A.-B., 1993. Numerical analysis of rolling for an aluminium at differ- 
ent temperatures. Proceedings of the international seminar MECAMAT'91 ISBN 9054103175.

Richelsen, A.-B., 1996. Comparison of a numerical analysis of rolling with experimental data. J. Mater. Processing Technology 57, 70-78.

Wei, Y., Hutchinson, J., 1997. Steady-State crack growth and work of fracture for solids characterized by strain gradient plasticity. J. Mech. Phys. Solids 45, 1253-1273.

Zienkiewicz, O., Jain, P., Onata, E., 1978. Flow of solids during forming and extrusion: some aspects of numerical solutions. Int. J. Solids. Structures 14, 15-38.

\section{Tables}

Table 1: Material properties (2H being the sheet thickness, see Fig. 1).

\begin{tabular}{lll}
\hline Parameter & Significance & Value \\
\hline$\sigma_{y} / E$ & Yield strain & 0.003 \\
$\nu$ & Poisson's ratio & 0.3 \\
$N$ & Strain hardening exponent & 0.1 \\
$m$ & Strain rate hardening exponent & $0.05-0.2$ \\
$\dot{\varepsilon}_{0}$ & Reference strain rate & 0.001 \\
$L_{D}$ & Dissipative length parameter & $0.05-0.5 \mathrm{H}$ \\
$L_{E}$ & Energetic length parameter & $0.05-0.5 \mathrm{H}$ \\
\hline
\end{tabular}




\section{Figures}

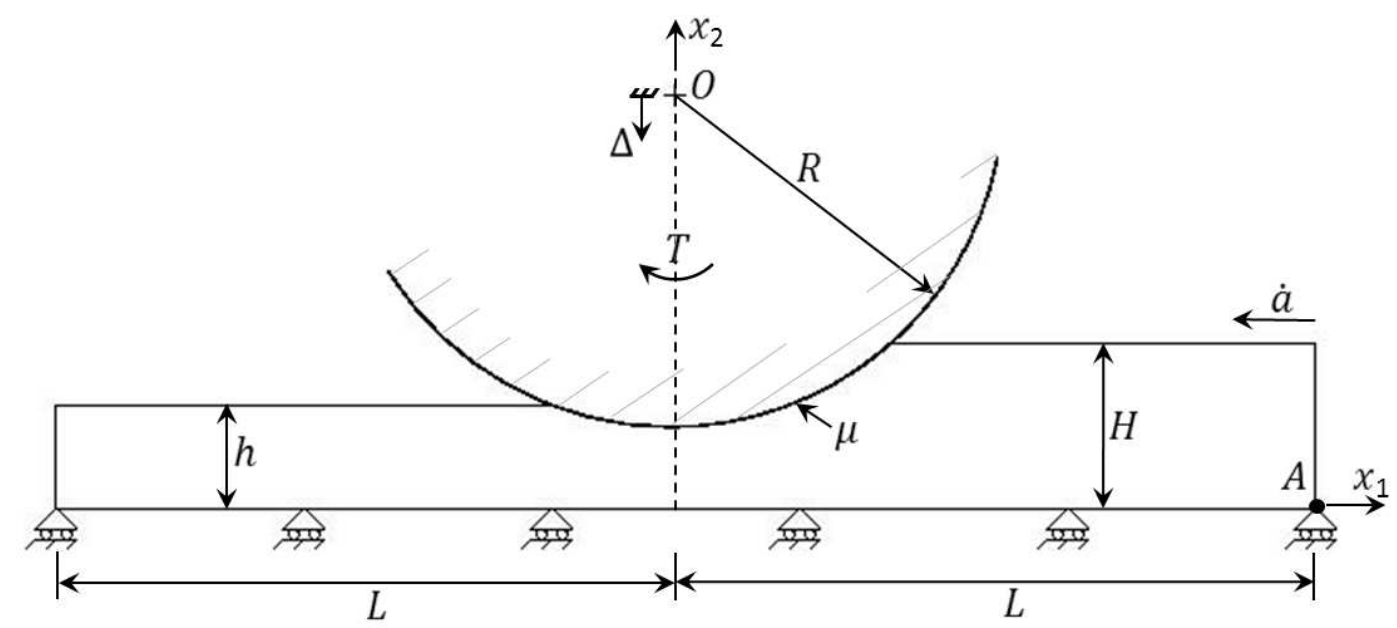

Figure 1: Parametrization of the rolling process under steady-state conditions, with symmetry applied at $x_{2}=0$. Throughout, $L / H=10$, with the domain discretized by equal sized squared elements of side length; $L^{(e)} / H=20$, and unit thickness. Not shown is the width of the sheet in the out-of-plane direction, $b$.
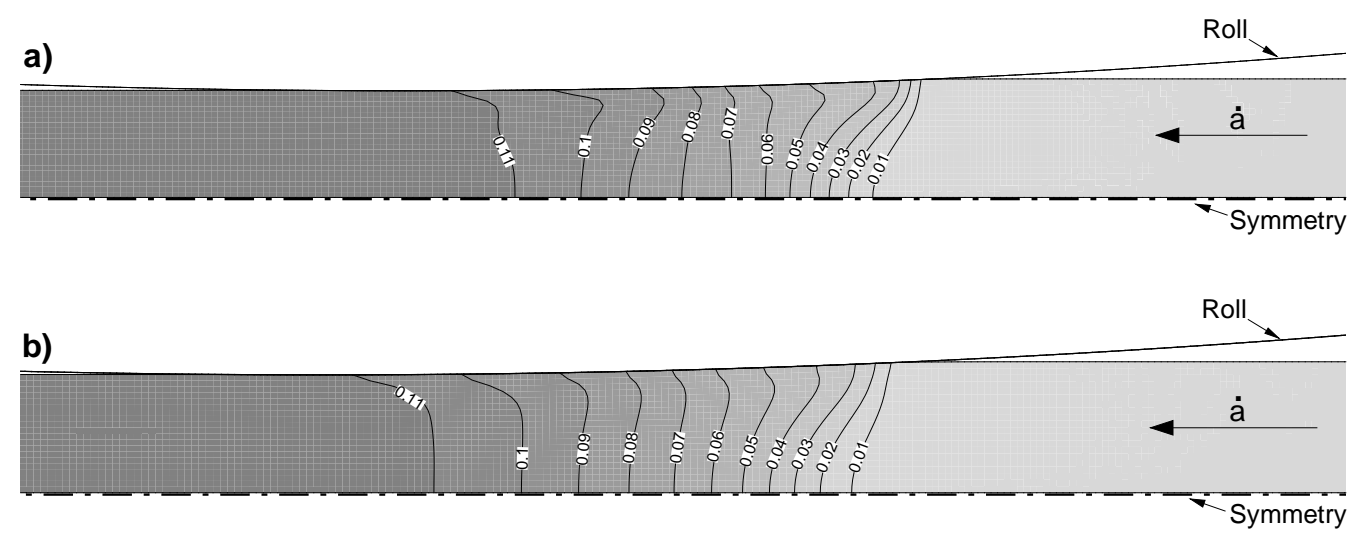

Figure 2: Effect of rate-sensitivity on plastic strain distribution for $L_{D} / H=0.05$ (close to the conventional limit, see e.g. Nielsen et al., 2015), with a) $m=0.05$ and b) $m=0.20$ for $\dot{a} /\left(\dot{\varepsilon}_{0} H\right)=100$. Here, showing curves of constant effective gradient enhanced plastic strain, $E^{p}$, for a fixed roller displacement of $\Delta / H=0.1\left(N=0.1, L_{E} / H=0, \sigma_{y} / E=0.003, R / H=100\right.$, and $\left.\mu=0.1\right)$. 


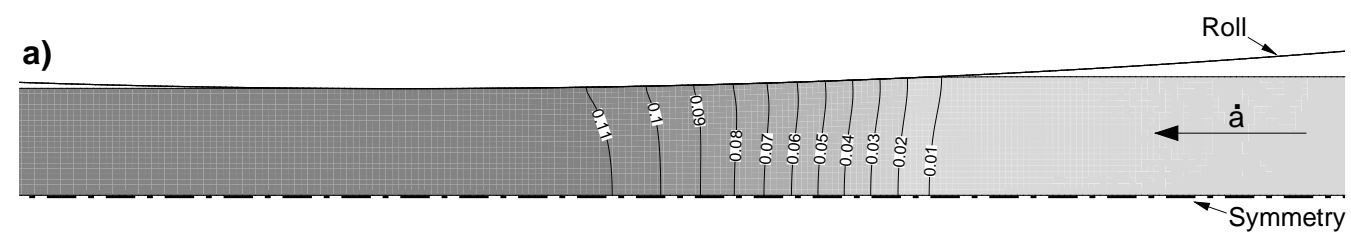

b)

Roll

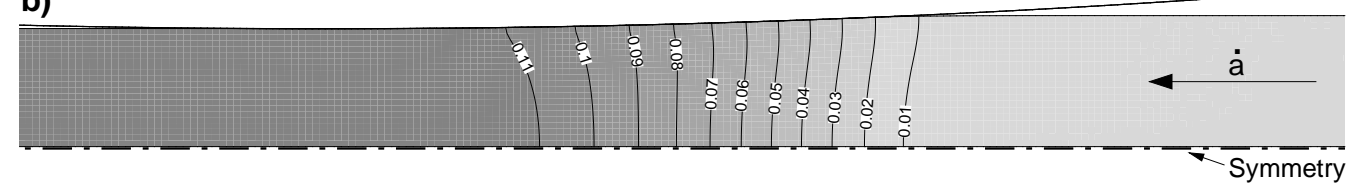

Figure 3: Effect of rate-sensitivity on plastic strain distribution for $L_{D} / H=0.50$ (rolling at small scale), with a) $m=0.05$ and b) $m=0.20$ for $\dot{a} /\left(\dot{\varepsilon}_{0} H\right)=100$. Here, showing curves of constant effective gradient enhanced plastic strain, $E^{p}$, for a fixed roller displacement of $\Delta / H=0.1(N=0.1$, $L_{E} / H=0, \sigma_{y} / E=0.003, R / H=100$, and $\left.\mu=0.1\right)$. 


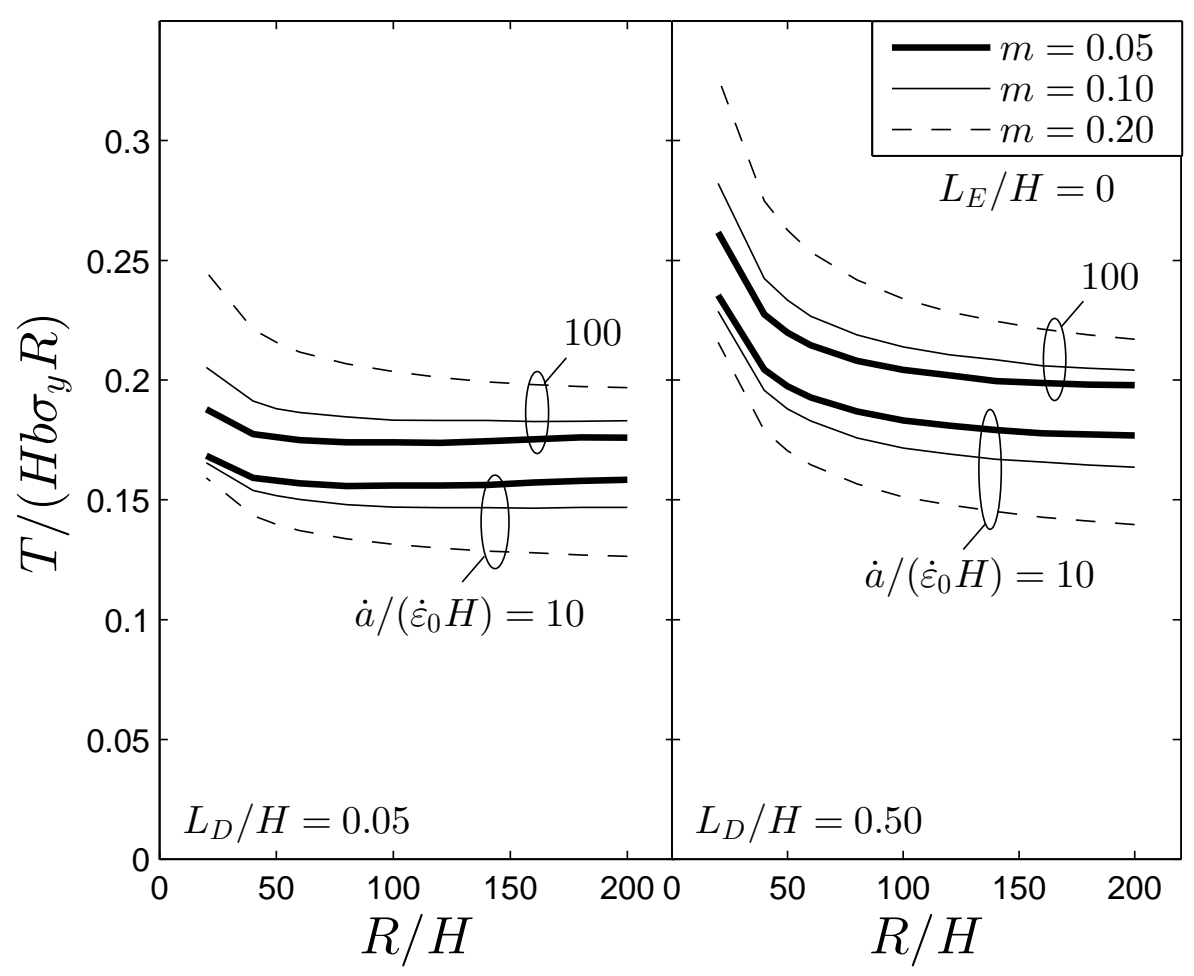

(a)

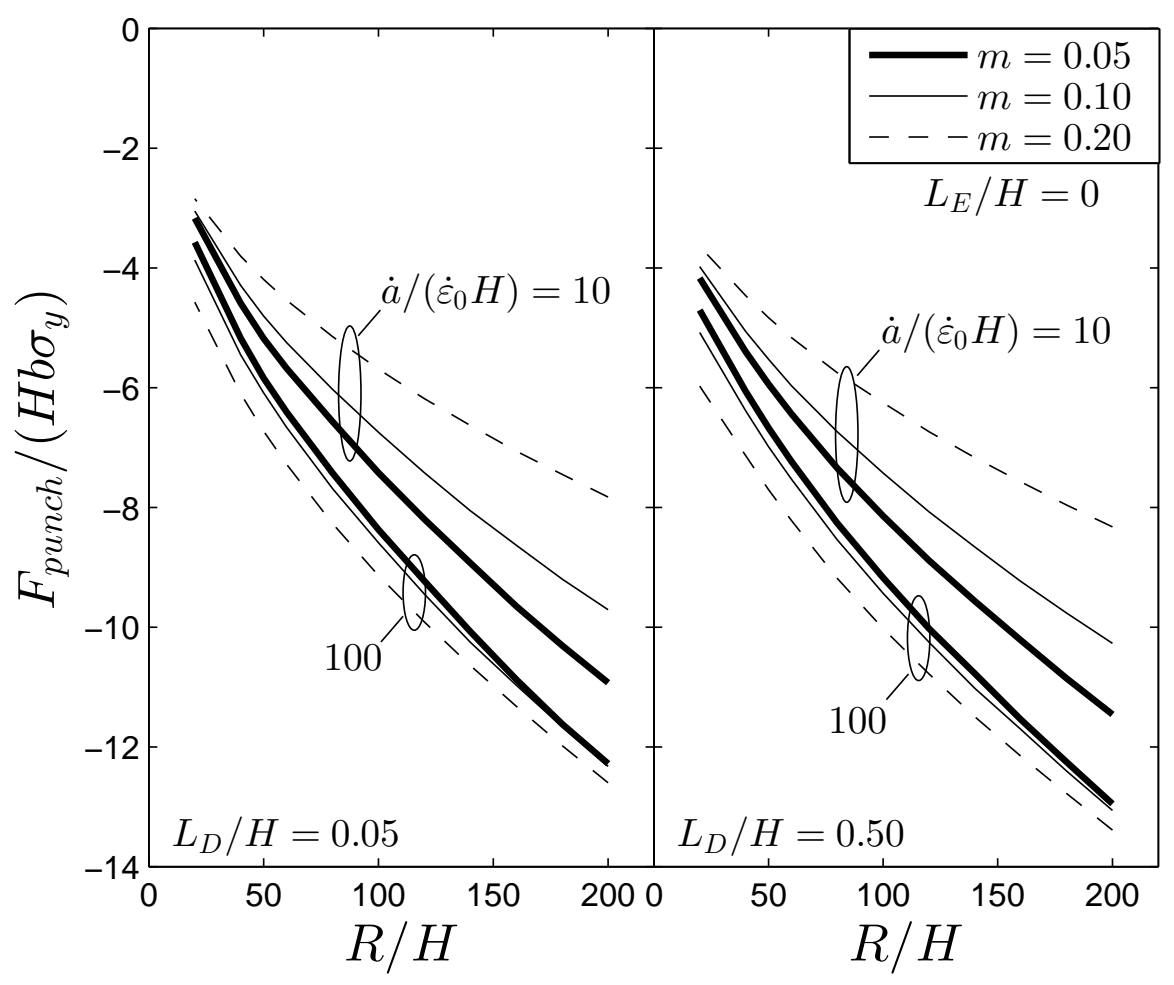

(b)

Figure 4: Rolling at two dissipative length scales (and $L_{E}=0$ ), for various roller radii. Here, showing a) applied roll torque, and b) applied punch force for a fixed roller displacement of $\Delta / H=0.1$, and a fast $\left(\dot{a} /\left(\dot{\varepsilon}_{0} H\right)=100\right)$ and slow $\left(\dot{a} /\left(\dot{\varepsilon}_{0} H\right)=10\right)$ moving sheet, respectively $\left(N=0.1, \sigma_{y} / E=0.003\right.$, and $\mu=0.1)$. The width of the out-of-plane direction is denoted, $b$. 


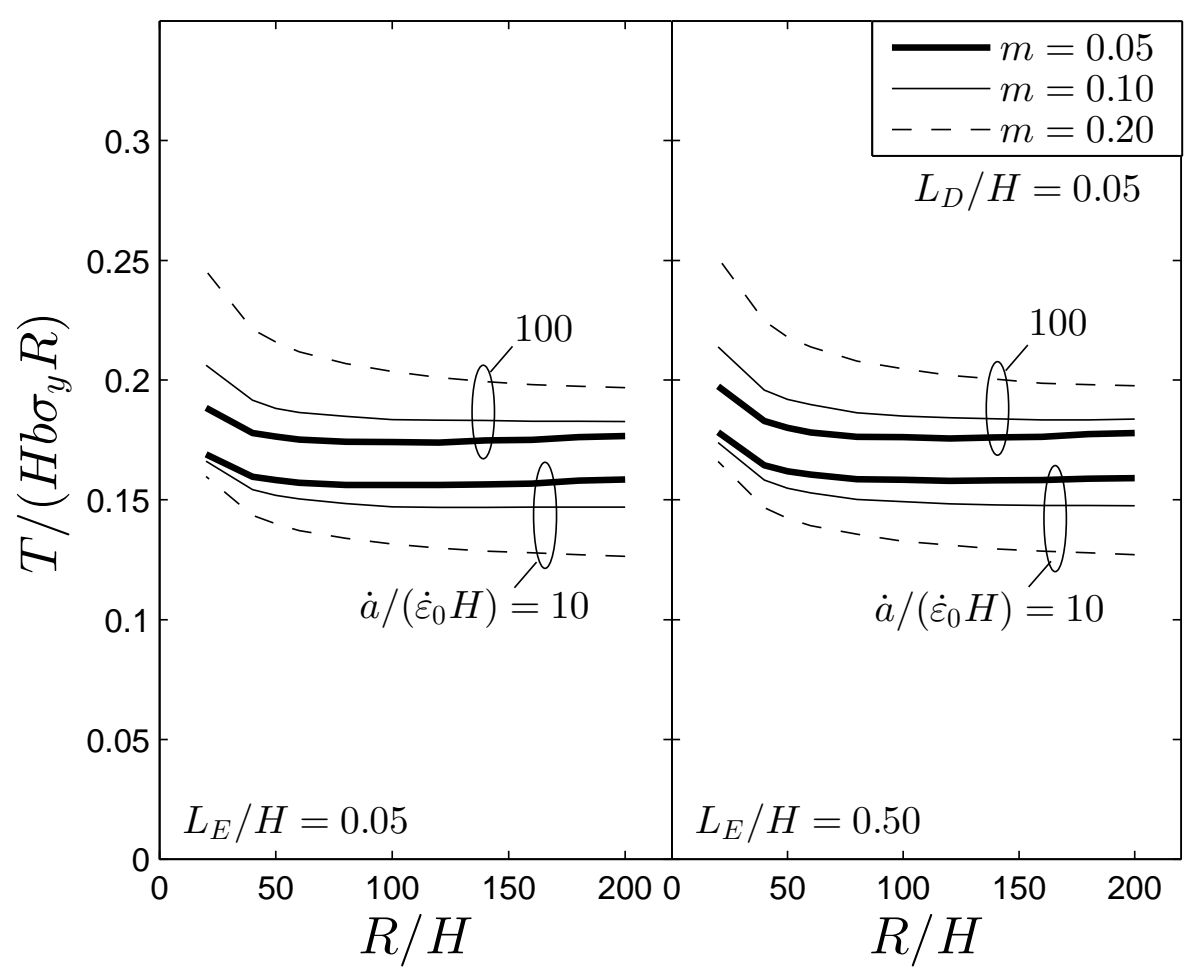

(a)

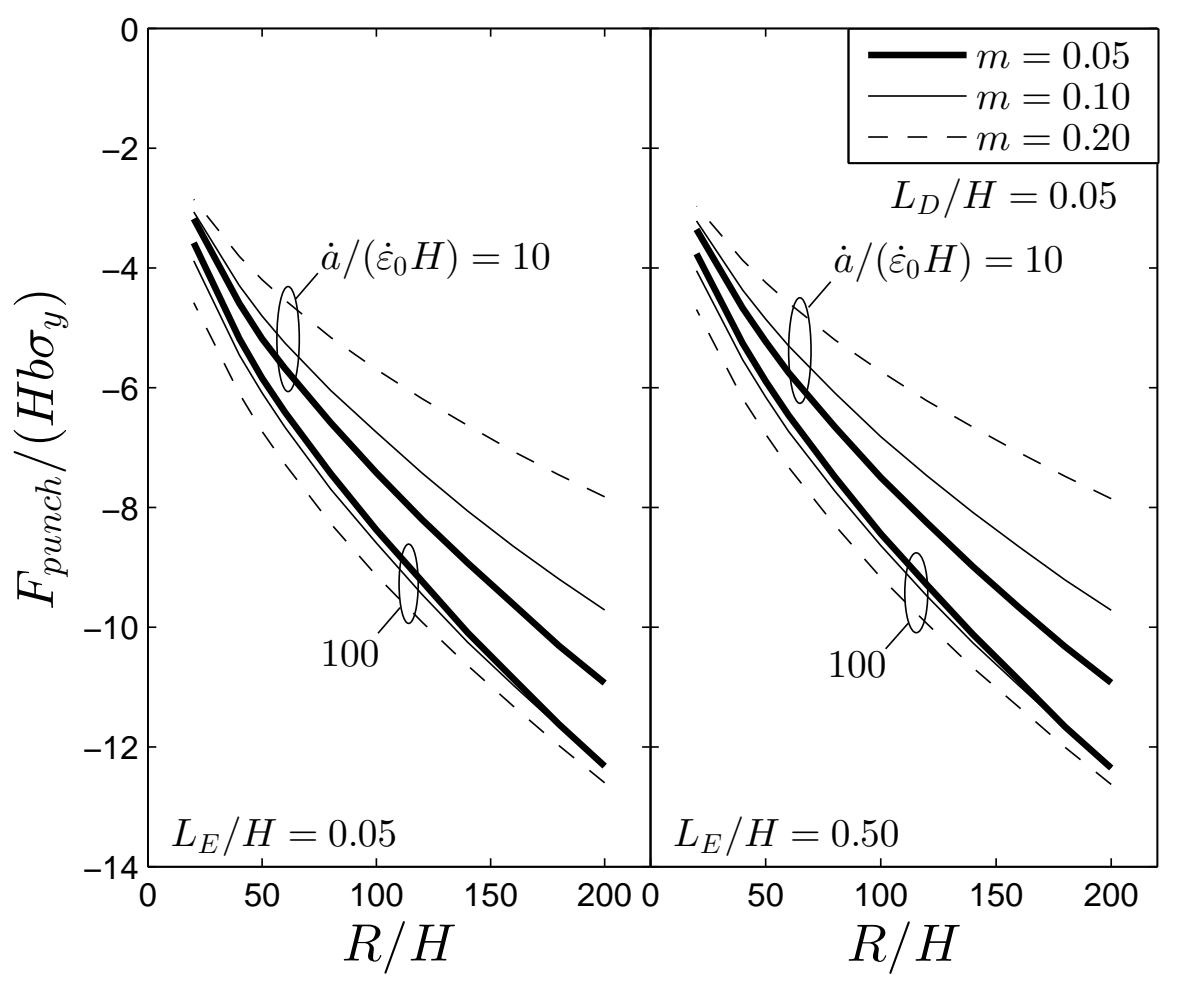

(b)

Figure 5: Rolling at two energetic length scales (and $L_{D} / H=0.05$ ), for various roller radii. Here, showing a) applied roll torque, and b) applied punch force for a fixed roller displacement of $\Delta / H=$ 0.1 , and a fast $\left(\dot{a} /\left(\dot{\varepsilon}_{0} H\right)=100\right)$ and slow $\left(\dot{a} /\left(\dot{\varepsilon}_{0} H\right)=10\right)$ moving sheet, respectively $(N=0.1$, $\sigma_{y} / E=0.003$, and $\mu=0.1$ ). The width of the out-of-plane direction is denoted, $b$. 

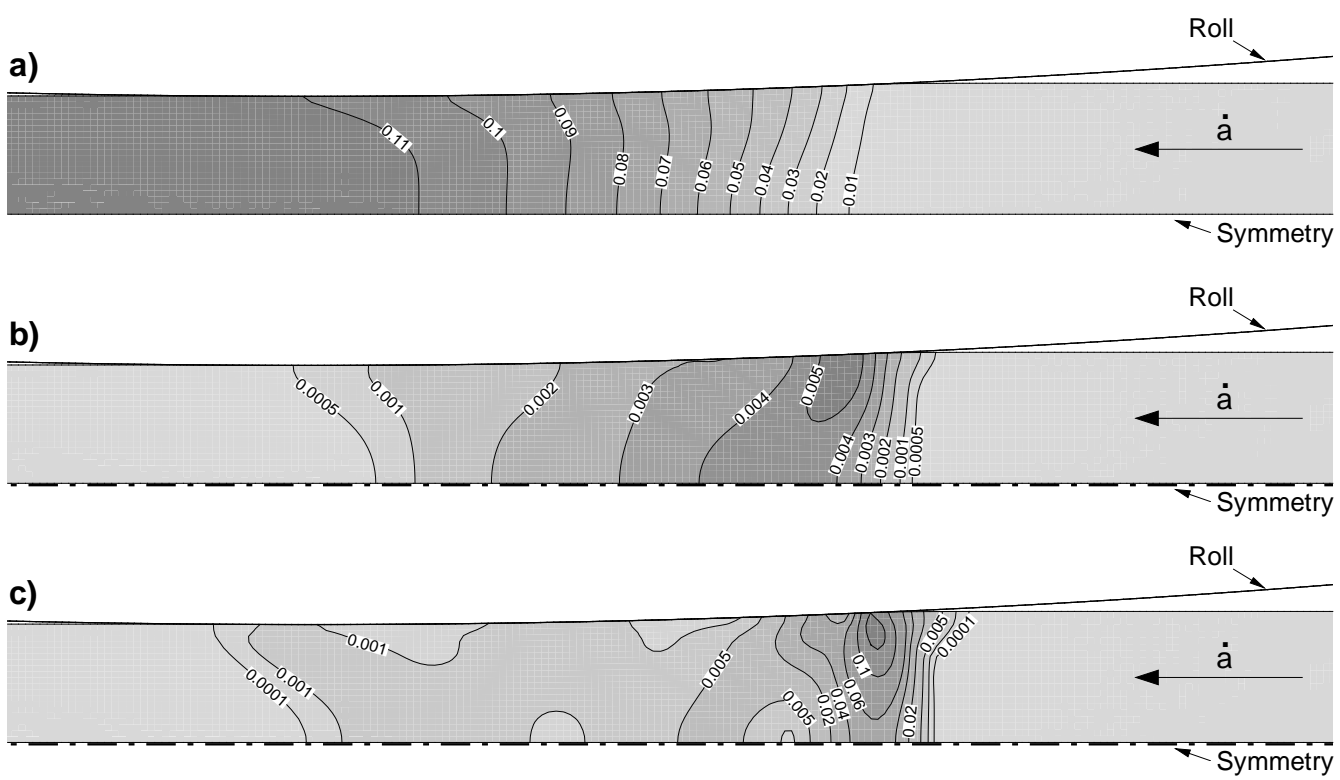

Figure 6: Effect the enegertic length parameter for $L_{E} / H=0.50$ (rolling at small scale), with $m=0.05$ and $\dot{a} /\left(\dot{\varepsilon}_{0} H\right)=100$. Here showing curves of constant a) effective gradient enhanced plastic strain, $E^{p}$, c) rate for the effective gradient enhanced plastic strain, $\dot{E}^{p}$, and c) Strain gradient contribution to the free energy; $\Psi_{G}=\frac{1}{2} G\left(L_{E}\right)^{2} \varepsilon_{i j, k}^{p} \varepsilon_{i j, k}^{p}$. All results are shown for a fixed roller displacement of $\Delta / H=0.1\left(N=0.1, L_{D} / H=0.05, \sigma_{y} / E=0.003, R / H=100\right.$, and $\left.\mu=0.1\right)$. 


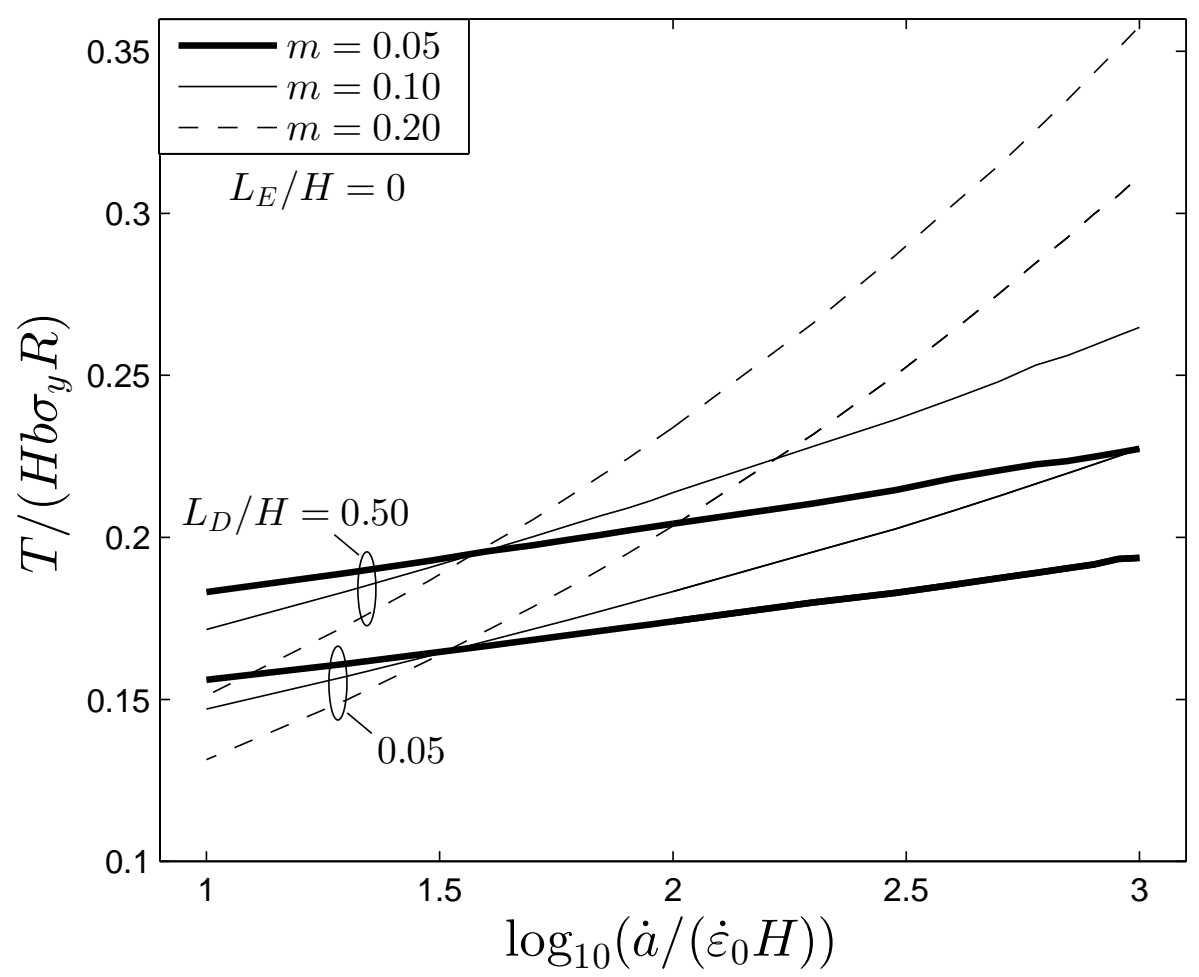

(a)

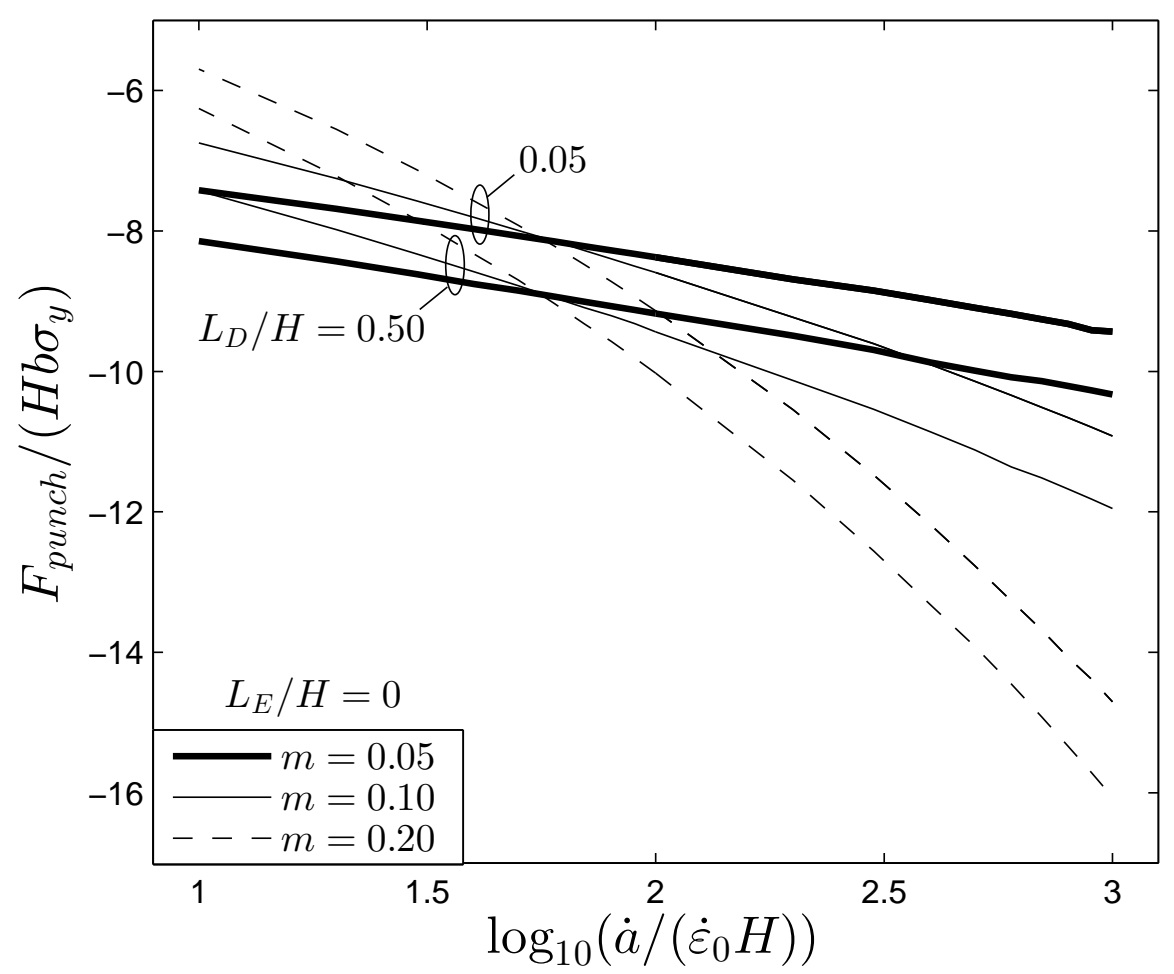

(b)

Figure 7: Effect of sheet velocity for different levels of rate-sensitivity, two distinct dissipative length scales $\left(L_{D} / H=[0.05,0.50]\right)$, and a fixed roller radius of $R / H=100$. Here, showing a) the applied torque, and b) the applied punch force, for a prescribed roller displacement of $\Delta / H=0.1(N=0.1$, $L_{E}=0, \sigma_{y} / E=0.003$, and $\left.\mu=0.1\right)$. The width of the out-of-plane direction is denoted, $b$. 


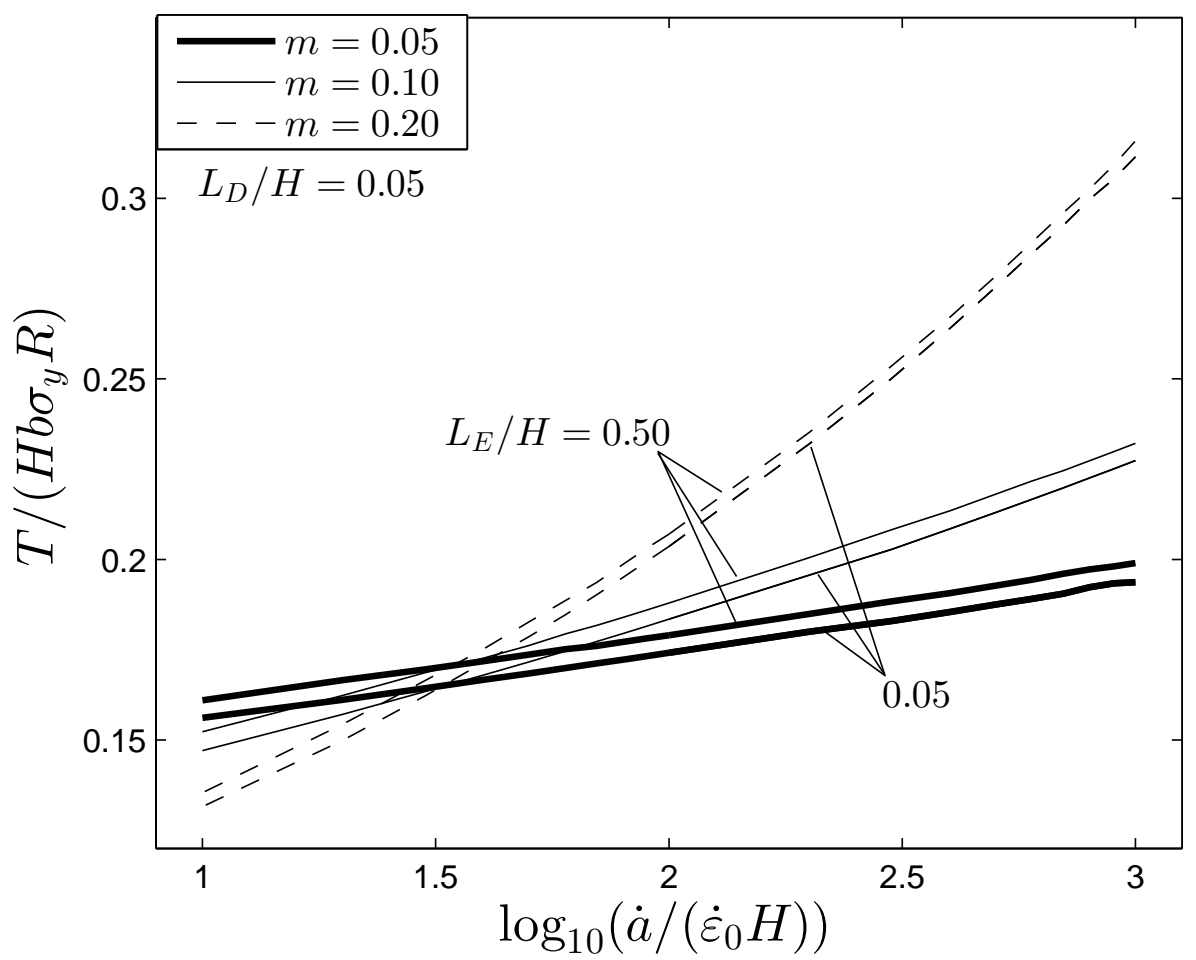

(a)

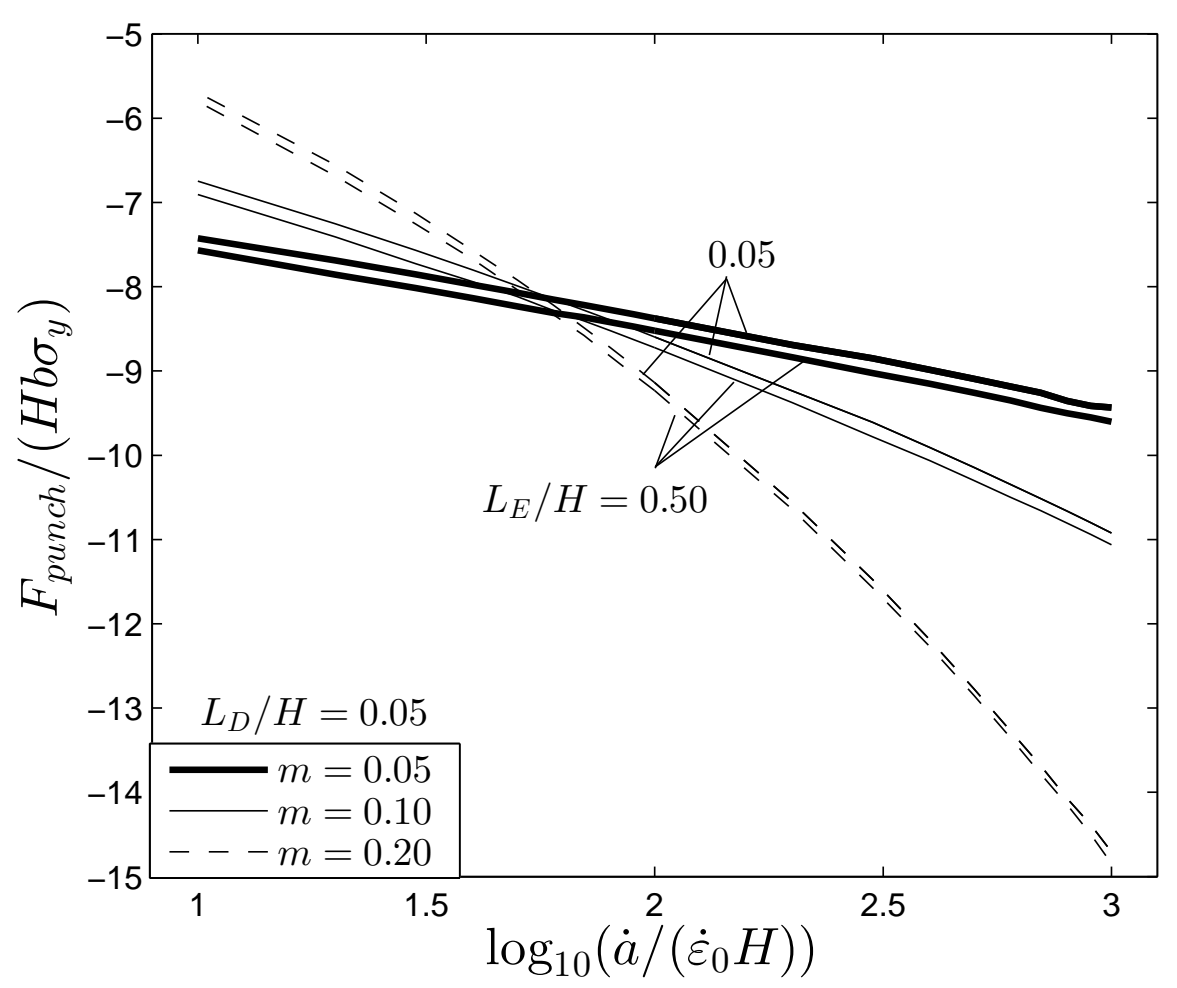

(b)

Figure 8: Effect of sheet velocity for different levels of rate-sensitivity, two distinct energetic length scales $\left(L_{E} / H=[0.05,0.50]\right)$, and a fixed roller radius of $R / H=100$. Here, showing a) the applied torque, and b) the applied punch force, for a prescribed roller displacement of $\Delta / H=0.1(N=0.1$, $L_{D} / H=0.05, \sigma_{y} / E=0.003$, and $\mu=0.1$ ). The width of the out-of-plane direction is denoted, $b$. 


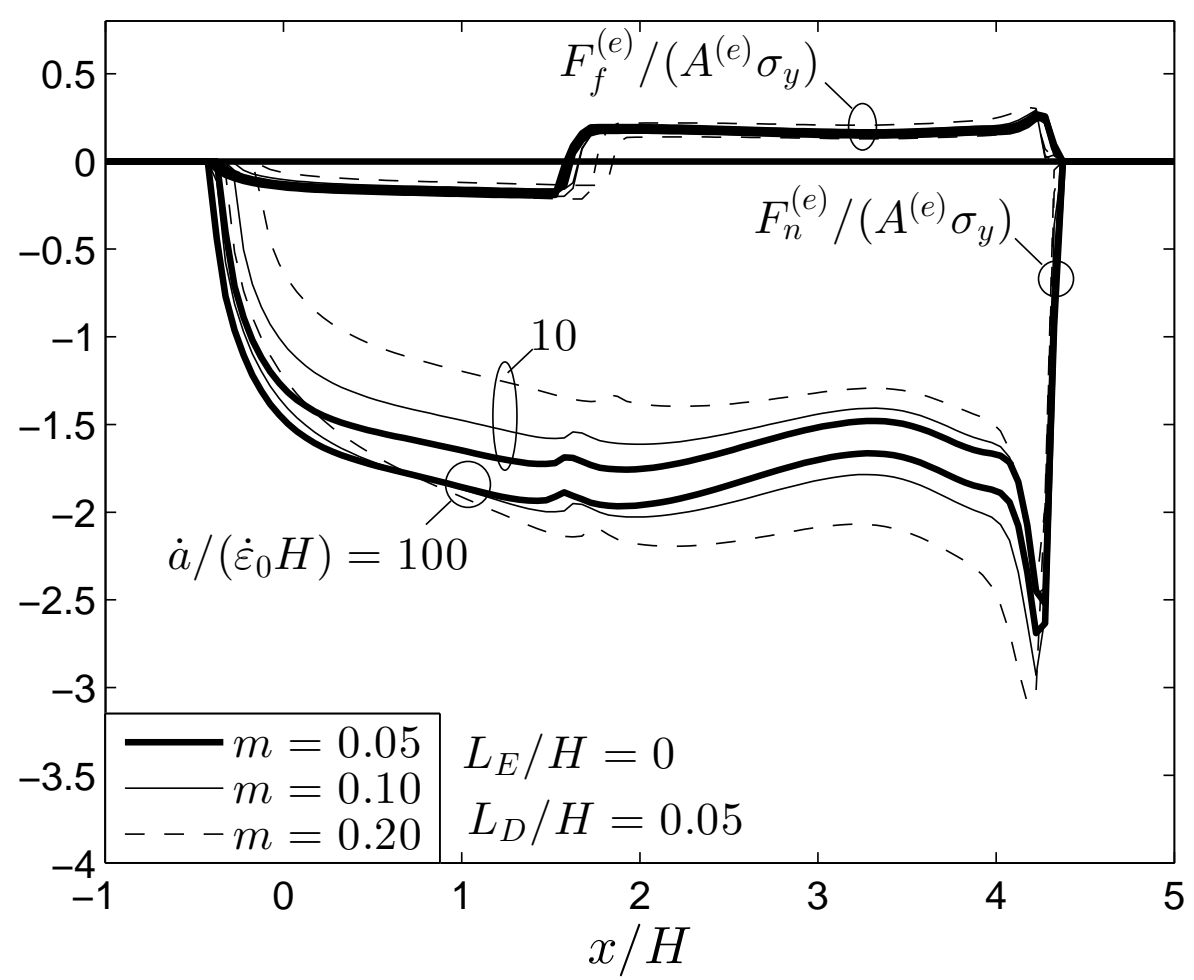

Figure 9: Contact conditions at the roller/sheet interface during rolling at large scale (nearing the conventional limit, $L_{E}=0$ and $L_{D} / H=0.05$ ). Here, showing the normalized friction forces (tangential traction) and normal forces (normal traction) for high/low sheet velocity and various rate-sensitivities $\left(N=0.1, \sigma_{y} / E=0.003, R / H=100, \Delta / H=0.1\right.$, and $\left.\mu=0.1\right)$. 


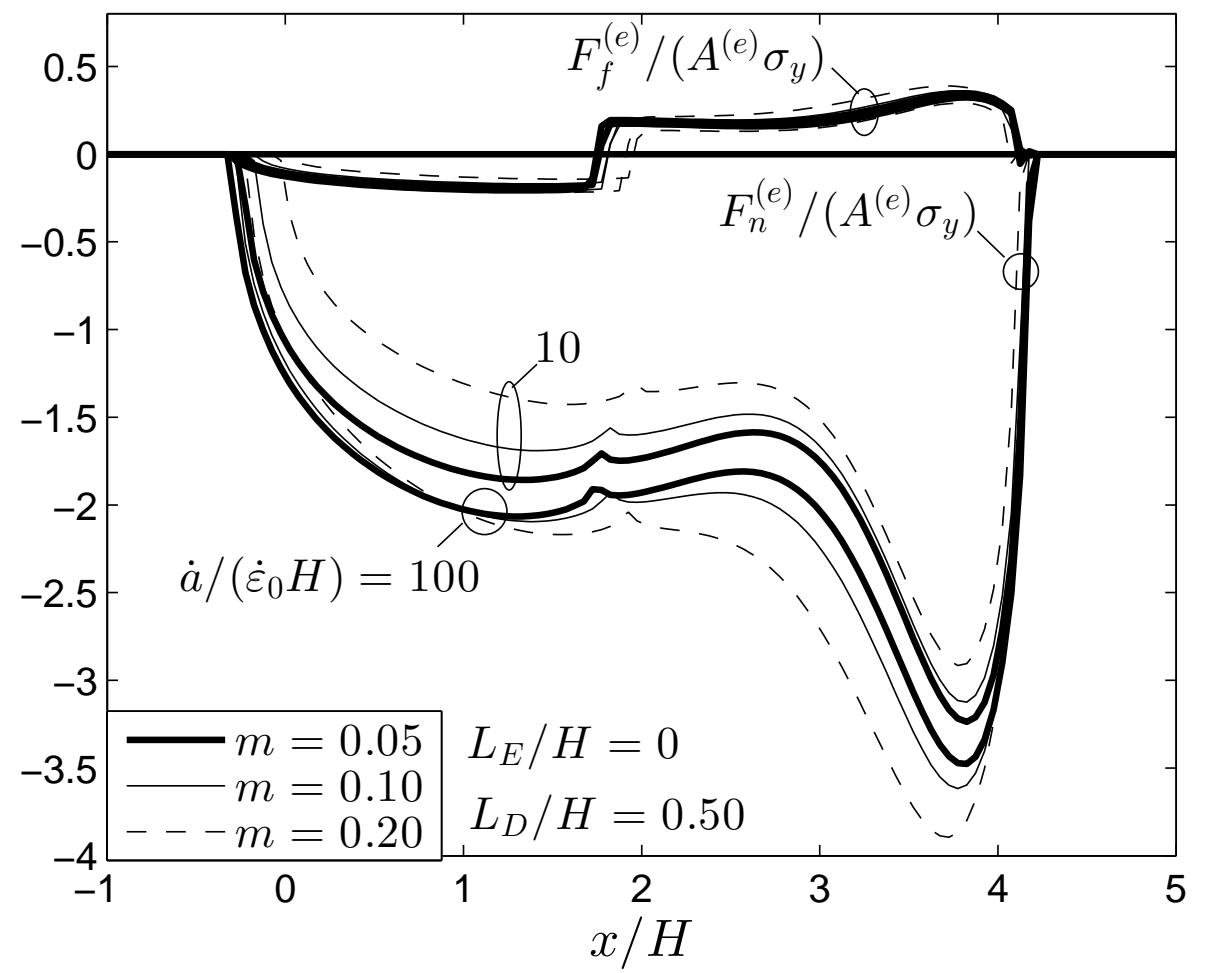

(a)

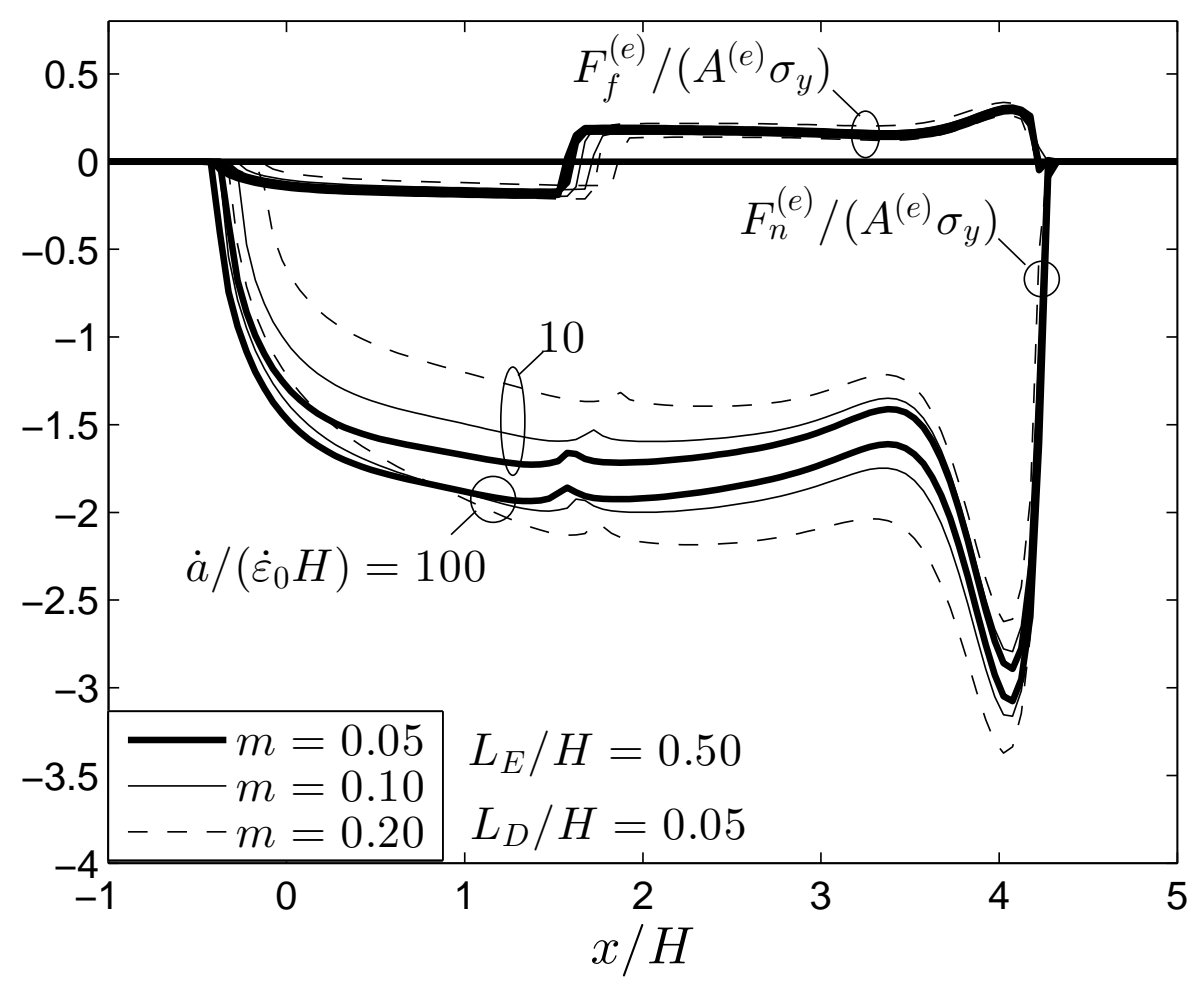

(b)

Figure 10: Contact conditions at the roller/sheet interface during rolling at small scale, either; a) small energetic length parameter $\left(L_{E} / H=0\right.$ and $\left.L_{D} / H=0.50\right)$, or b) small dissipative length parameter $\left(L_{E} / H=0.50\right.$ and $\left.L_{D} / H=0.05\right)$. Here, showing the normalized friction forces (tangential traction) and normal forces (normal traction) for high/low sheet velocity and various rate-sensitivities $(N=0.1$, $\sigma_{y} / E=0.003, R / H=100, \Delta / H=0.1$, and $\left.\mu=89^{1}\right)$. 